TRANSACTIONS OF THE

AMERICAN MATHEMATICAL SOCIFTY

Volume 281. Number 2. Fehruary 1984

\title{
SUPERCOMPACTNESS OF COMPACTIFICATIONS AND HYPERSPACES
}

\author{
BY \\ MURRAY G. BELL'
}

\begin{abstract}
We prove a theorem which implies that if $\gamma \omega$ is a supercompact compactification of the countable discrete space $\omega$, then $\gamma \omega-\omega$ is separable. This improves an earlier result of the author's that such a $\gamma \omega$ must have $\gamma \omega-\omega \mathrm{ccc}$.

We prove a theorem which implies that the hyperspace of closed subsets of $2^{\omega_{2}}$ is not a continuous image of a supercompact space. This improves an earlier result of L. Sapiro that the hyperspace of closed subsets of $2^{\omega_{2}}$ is not dyadic.
\end{abstract}

1. Introduction. A space $X$ is supercompact, de Groot [9], if it possesses an open subbase $\delta$ such that every open cover of $X$ from $\delta$ has a 2 subcover. The vague finite of compactness is replaced by the concrete two of supercompactness. Many compact spaces are supercompact, see van Mill [11], but not all.

We had earlier proved [2] that if $\gamma X$ was a supercompactification of a locally compact space $X$, then any collection of disjoint open sets of $\gamma X-X$ had size at most the weight of $X$. In $\S 3$, we prove a stronger theorem via a simpler proof. Namely, that if $\gamma X$ is a supercompactification of a locally compact space $X$, then $\gamma X-X$ has a dense subspace of size at most the weight of $X$.

The remaining sections are devoted to the proof of Theorem 6.1, which implies that the hyperspace of $2^{\omega_{2}}$ is not the continuous image of a supercompact space. We mention that all powers of 2 are supercompact spaces. It is well known that $\exp 2^{\omega}$ is homeomorphic to $2^{\omega}$. Sirota [15] proved that $\exp 2^{\omega_{1}}$ is homeomorphic to $2^{\omega_{1}}$. Šapiro [14] proved that not only was $\exp 2^{\omega_{2}}$ not homeomorphic to $2^{\omega_{2}}$, it was not even dyadic. We acknowledge a debt to the paper of Šapiro. It suggested to us the Subbase Lemma of $\S 4$, which is of independent interest, as well as the line of attack towards our generalization.

2. Notation and definitions. For a cardinal $\kappa, \kappa^{+}$is the successor cardinal and $2^{\kappa}=\{f: f$ is a function from $\kappa$ to 2$\}$. If $H \subseteq 2^{\kappa}$ and $\alpha<\kappa$, then $H \backslash \alpha$ denotes $\{f \backslash \alpha: f \in H\}$. If $\delta$ is a collection of sets and $S$ is a set, then $\delta^{n}$ denotes $\left\{\cap \delta^{\prime}: \delta^{\prime} \subseteq \delta\right\}$ and $\delta \uparrow S$ denotes $\{T \cap S: T \in \delta\}$. $\delta$ is linked if all 2-fold intersections of members of $\delta$ are nonempty. $\delta$ is centered if all finite intersections of

\footnotetext{
Received by the editors November 17, 1982.

1980 Mathematics Subject Classification. Primary 54D30; Secondary 54B20.

Key words and phrases. Supercompact, hyperspace, dyadic.

${ }^{1}$ This research was supported by Grant No. U0070 from the Natural Sciences and Engineering Research Council of Canada.
} 
members of $\delta$ are nonempty. $\delta$ is $\kappa$-centered if $\delta$ is the union of $\kappa$ many centered subcollections. $\delta$ is binary if all linked subcollections of $\delta$ have a nonempty intersection.

We assume that all of our spaces are completely regular and Hausdorff. If $Y$ is a continuous image of $X$, then we write $X \rightarrow Y$. A $G_{\kappa}$ subset of a space $X$ is a subset that is an intersection of at most $\kappa$ open subsets of $X$. The collection of all nonempty open subsets of $X$ is denoted by $t(X) . X$ is said to be $\kappa$-centered if $t(X)$ is $\kappa$-centered. We will use the following standard cardinal functions and refer the reader to Juhász [10]: Cardinality $|X|$, weight $w(X)$, net weight $n w(X)$, density $d(X)$, spread $s(X)$ and cellularity $c(X)$. If $c(X) \leqslant \omega$, then we say that $X$ is ccc.

As a space, $2^{\kappa}$ is endowed with the product topology. The Alexandroff one-point compactification of the discrete space $\kappa$ is denoted by $\alpha \kappa$ and the Stone-Čech compactification of the discrete space $\kappa$ is denoted by $\beta \kappa$. exp $X$ is the hyperspace of all closed subsets of $X$ endowed with the topology which has an open base consisting of all sets of the form $\left\langle O_{1}, \ldots, O_{n}\right\rangle=\left\{F: F\right.$ is a closed subset of $X, F \subseteq \cup_{i=1}^{n} O_{i}$ and for all $\left.i \leqslant n, F \cap O_{i} \neq \varnothing\right\}$ where the $O_{i}$ 's are open subsets of $X$. It is well known, Vietoris [16], that exp $X$ is compact iff $X$ is compact.

A space is dyadic, Alexandroff [1], if it is a continuous image of a power of 2 . A space is supercompact, de Groot [9], if it possesses a binary closed subbase. If $\lesssim$ is a binary closed subbase for $X$, then $\varsigma^{n}$ is also a binary closed subbase for $X$. Continuous images of supercompact spaces will be called superadic spaces. Indeed, there are superadic spaces that are not supercompact, van Mill and Mills [12]. Since a power of 2 is supercompact, superadic spaces are a generalization of dyadic spaces. In fact, they generalize the $m$-adic and $\xi$-adic spaces of Mrówka [13]. Another interesting generalization of dyadic spaces are the hyadic spaces of van Douwen [6], i.e., continuous images of hyperspaces of compact spaces. Both the superadic spaces, van Douwen and van Mill [7], and the hyadic spaces, van Douwen [6], have nontrivial convergent sequences. I do not know whether all supercompact (and therefore superadic) spaces are hyadic.

3. Superadic compactifications. In this section, $\gamma X$ represents a compactification of $X$. A collection of sets $\mathcal{S}$ is said to be $r$-centered in a set $S$ if $\mathcal{S}=\cup_{i<r} \mathcal{S}_{i}$ where for each $i<r,\left\{S \cap T: T \in \mathcal{S}_{i}\right\}$ is a centered collection.

\subsection{TheOREM. If $\gamma X$ is superadic, then $\gamma X-X$ is $n w(X)$-centered.}

Proof. Let $f$ map $Y$ continuously onto $\gamma X$ and let $\delta=\mathcal{S}^{\cap}$ be a binary closed subbase for $Y$. Let $\mathscr{N}$ be a network for $X$ of size $n w(X)$ closed under finite unions and such that $\varnothing \notin \Re$. Put $\mathscr{Q}=\{U: U$ is open in $\gamma X$ and $U \nsubseteq X\}$. Choose $W \subseteq Y$ of size at most $d(X)$ such that $f(W)$ is a dense subspace of $X$.

For each $U \in \mathcal{Q}$ and for each $w \in W$, set $\mathcal{S}(U, w)=\{S \in \mathcal{S}: w \in S$ and $f(S) \not \subset$ $U\}$. For each $w \in W$ and for each $N \in \mathfrak{N}$ set $\mathscr{Q}(w, N)=\{U \in \mathcal{Q}:$ there exists $S \in \mathcal{S}$ and there exists a finite subset $F$ of $Y-f^{-1}(f(S))$ such that $w \in S \subseteq f^{-1}(U)$, $f(S) \not X, f(F) \cap X \subseteq N \subseteq X-f(S)$ and for all $T \in \mathcal{S}(U, w), T \cap F \neq \varnothing\}$.

Claim 1. For each $w \in W$ and for each $N \in \mathfrak{R}$, there exists an $r<\omega$ such that $\mathcal{Q}(w, N)$ is $r$-centered in $\gamma X-X$. 
Proof of Claim. If $\mathcal{U}(w, N)$ is 1 -centered in $\gamma X-X$, then let $r=1$. If not, then there exists a finite $\mathscr{F} \subseteq \mathcal{Q}(w, N)$ such that $\cap \mathscr{F} \subseteq X$. For each $U \in \mathscr{F}$, let $S_{u}$ and $F_{u}$ witness the fact that $U \in \mathcal{Q}(w, N)$. Then, every $V \in \mathcal{U}(w, N)$ contains an element of $\cup_{U \in \mathscr{F}} f\left(F_{u}\right) \cap(\gamma X-X)$, so we can let $r=\left|\cup_{U \in \mathscr{F}} f\left(F_{u}\right) \cap(\gamma X-X)\right|$. For, if $V \in \mathcal{Q}(w, N)$, then let $S_{v}$ and $F_{v}$ witness this fact. Since $f\left(S_{v}\right) \not X$, we have $f\left(S_{v}\right) \nsubseteq \cap \mathscr{F}$, therefore, there exists $U \in \mathscr{F}$ such that $f\left(S_{v}\right) \not U$. Hence, $S_{v} \in \mathcal{S}(U, w)$ and because $U \in \mathcal{Q}(w, N)$ we have $S_{v} \cap F_{u} \neq \varnothing$. So, $f\left(S_{v} \cap F_{u}\right) \neq \varnothing$. If $f\left(S_{v} \cap F_{u}\right) \subseteq X$, then $f\left(S_{v} \cap F_{u}\right) \subseteq f\left(F_{u}\right) \cap X \subseteq N \subseteq X-f\left(S_{v}\right)$, contradiction. Therefore, $f\left(S_{v} \cap F_{u}\right) \cap(\gamma X-X) \neq \varnothing$ and so $f\left(S_{v}\right) \cap f\left(F_{u}\right) \cap(\gamma X-X) \neq \varnothing$. Since $f\left(S_{v}\right) \subseteq V$, we have $V \cap f\left(F_{u}\right) \cap(\gamma X-X) \neq \varnothing$.

Claim 2. $\mathscr{U}=\cup\{\mathscr{U}(w, N): w \in W$ and $N \in \mathfrak{N}\}$.

Proof of Claim. Let $U \in \mathcal{Q}$. Pick $x \in U-X$. By regularity, there exists an open subset $O$ of $\gamma X$ such that $x \in O \subseteq \bar{O} \subseteq U$. Therefore, $f^{-1}(x) \subseteq f^{-1}(O) \subseteq f^{-1}(\bar{O}) \subseteq$ $f^{-1}(U)$. There exists a finite $\varsigma^{\prime} \subseteq \S$ such that $f^{-1}(\bar{O}) \subseteq \cup \varsigma^{\prime} \subseteq f^{-1}(U)$. Since $f$ is a closed map and $f(W)$ is dense in $X$, there must exist an $S_{u} \in \mathcal{S}^{\prime}$ such that $S_{u} \cap f^{-1}(x) \neq \varnothing$ and $S_{u} \cap W \neq \varnothing$. Pick $w \in S_{u} \cap W$. Note that since $x \in f\left(S_{u}\right)$, $f\left(S_{u}\right) \nsubseteq X$.

Since $Y-f^{-1}(U) \subseteq Y-f^{-1}\left(f\left(S_{u}\right)\right)$, there exists $\left\{S_{i}: i<m\right\} \subseteq \delta$ such that $Y-$ $f^{-1}(U) \subseteq \cup_{i<m} S_{i} \subseteq Y-f^{-1}\left(f\left(S_{u}\right)\right)$. For each $i<m$, set $\delta_{i}=\{S \in \delta(U, w): S \cap$ $\left.S_{i} \neq \varnothing\right\}$. Then, $\delta(U, w)=\cup_{i<m} \delta_{i}$. For each $i<m, \delta_{i} \cup\left\{S_{i}\right\}$ is linked. Hence, there exists $x_{i} \in \cap \mathcal{S}_{i} \cap S_{i} \subseteq Y-f^{-1}\left(f\left(S_{u}\right)\right)$. Set $F_{u}=\left\{x_{i}: i<m\right\}$. Since $f\left(F_{u}\right) \cap$ $X \subseteq X-f\left(S_{u}\right)$, there exists $N \in \Re$ such that $f\left(F_{u}\right) \cap X \subseteq N \subseteq X-f\left(S_{u}\right)$. Finally, $S_{u}$ and $F_{u}$ witness the fact that $U \in \mathcal{Q}(w, N)$.

From Claims 1 and 2, it follows that

$$
t(\gamma X-X)=\cup\{\{U \cap(\gamma X-X): U \in \mathcal{Q}(w, N)\}: w \in W \text { and } N \in \mathscr{T}\}
$$

where each of these collections is $r$-centered for some $r<\omega$. Since $|W|=d(X) \leqslant$ $n w(X)$, we get that $\gamma X-X$ is $n w(X)$-centered.

REMARK 1 . In the theorem, if $X$ is nowhere locally compact, then the conclusion is true regardless of whether $\gamma X$ is superadic or not. The theorem has nontrivial content for somewhere locally compact $X$ 's.

3.2. Corollary. If $X$ is locally compact and $\gamma X$ is superadic, then $d(\gamma X-X) \leqslant$ $n \omega(X)$. In particular, if $\gamma \omega$ is superadic, then $\gamma \omega-\omega$ is separable.

Proof. From Theorem 3.1 we conclude that $\gamma X-X$ is $n w(X)$-centered. Since $X$ is locally compact, $\gamma X-X$ is compact and hence $d(\gamma X-X) \leqslant n w(X)$. Note that $n w(X)=w(X)$ for locally compact spaces $X$.

REMARK 2. This corollary improves an earlier result of the author [2], that if $\gamma \omega$ is superadic, then $\gamma \omega-\omega$ is ccc. The earlier proof had an unnecessary use of infinitary combinatorics. The present proof is elementary. So elementary, in fact, that if one follows through the proof of Theorem 3.1, with $X=\omega, Y=\gamma \omega, f=$ identity, $\Re=\{F: F$ is a nonempty finite subset of $\omega\}$ and $W=\omega$, then one gets a proof without the aid of the Axiom of Choice of the following: (ZF) If $\gamma \omega$ is supercompact, then $t(\gamma w-w)$ is the union of countably many subcollections each of which is 
$r$-centered for some $r<\omega$. Since there is in ZF a compactification $\gamma \omega$ for which $\gamma \omega-\omega$ has $c$ isolated points, for example, Example 1.4 in [7], and $c$ is not the union of countably many finite sets, we get that ZF alone implies that not all compact Hausdorff spaces are supercompact.

REMARK 3. In [3], compactifications $\gamma \omega$ are constructed for which $\gamma \omega-\omega$ is ccc but not separable. By the theorem, none of these compactifications are superadic. However, they can be constructed to have arbitrary compactness number $>2$, see [4]. The compactness number of a compact space $X, \operatorname{cmpn}(X)$, is the least $n<\omega$, if one exists, such that $X$ possesses an $n$-ary closed subbase, Bell and van Mill [5]. If no such $n<\omega$ exists, then one says $\operatorname{cmpn}(X)=\infty$.

No results similar to the theorem or corollary exist for compactness number $>2$. There is a compactification $\gamma \omega$ such that cmpn $\gamma \omega=3$ and $\gamma \omega-\omega$ is not ccc, cf. van Douwen and van Mill [7].

3.3. Corollary. If $\exp (\gamma X)$ is superadic, then $\gamma X-X$ is $n w(X)$-centered.

Proof. Set $X^{*}=\{F: F$ is a finite subset of $X\}$. Then $X^{*}$ is a dense subspace of $\exp (\gamma X)$. Furthermore, $n w\left(X^{*}\right)=n w(X)$. By our theorem, $\exp (\gamma X)-X^{*}$ is $n w(X)$-centered. In particular,

$$
\left\{\langle O\rangle-X^{*}: O \in t(\gamma X)\right\}-\{\phi\}=\cup\left\{\mathfrak{C}_{\alpha}: \alpha<n w(X)\right\}
$$

where each $\hat{\theta}_{\alpha}$ is centered. For each $\alpha<n w(X)$, set $\theta_{\alpha}^{\prime}=\{O-X: O \in t(X)$ and $\left.\langle O\rangle-X^{*} \in \theta_{\alpha}\right\}$. Thus, $t(\gamma X-X)=\cup\left\{\theta_{\alpha}^{\prime}: \alpha<n w(X)\right\}$ and each $\Theta_{\alpha}^{\prime}$ is centered.

3.4. EXAmple. A 0-dimensional space $X$ with $w(X)=\omega_{1}$ and exp $X$ not superadic: Let $X$ be any compactification $\gamma \omega$ of $\omega$ of weight $\omega_{1}$ and having $\gamma \omega-\omega$ homeomorphic to $\alpha \omega_{1}$.

3.5. ExAmple. A 0 -dimensional space $X$ with $\exp X$ superadic but not dyadic: Let $\kappa$ be an uncountable cardinal and set $X=\alpha \kappa$. $\exp X$ is not dyadic because $\exp X$ is not ccc. We will show that $\exp X$ is supercompact by producing a binary closed subbase for $\exp X$. Set $\mathcal{Q}=\{\langle\{\gamma\}\rangle: \gamma<\kappa\} \cup\{\langle X-\{\gamma\}\rangle: \gamma<\kappa\}$. It is easily checked that $\mathscr{Q} \cup\{\exp X-A: A \in \mathbb{Q}\} \cup\{\{F\}: F$ is a finite subset of $\kappa\}$ is a binary closed subbase for $\exp X$. Note that superadicity of $\exp X$ places no weight restrictions on $X$. This contrasts with Sapiro's result [13] that dyadicity of $\exp X$ implies $w(X) \leqslant c$.

4. The Subbase Lemma. The following general result on subbases plays a key role in our main theorem of $\S 6$.

4.1. The Subbase Lemma. Let $\delta=\varsigma^{n}$ be a closed subbase for a compact space $X$. Let $f$ be a continuous map from $X$ onto a space $Y$. If $F$ is a nonempty closed $G_{\kappa}$ subset of $Y$, then there exists a nonempty $S \in \mathcal{s}$ such that $f(S) \subseteq F$ and $f(S)$ is a $G_{\kappa}$ subset of $Y$.

Proof. Let $F=\bigcap_{\alpha<\kappa} O_{\alpha}$ where each $O_{\alpha}$ is open in $Y$. For each $\alpha<\kappa$, choose a finite set $\left\{S_{i}^{\alpha}: i<n_{\alpha}\right\} \subseteq$ S such that $f^{-1}(F) \subseteq \cup_{i<n_{\alpha}} S_{i}^{\alpha} \subseteq f^{-1}\left(O_{\alpha}\right)$. Then,

$$
f^{-1}(F)=\bigcap_{\alpha<\kappa}\left(\bigcup_{i<n_{\alpha}} S_{i}^{\alpha}\right)=\bigcup\left\{\bigcap_{\alpha<\kappa} S_{\varphi(\alpha)}^{\alpha}: \varphi \in \prod_{\alpha<\kappa} n_{\alpha}\right\} .
$$


Since $\delta=\varsigma^{\cap}$, each $\cap_{\alpha<\kappa} S_{\varphi(\alpha)}^{\alpha} \in \mathcal{S}$. Give each $\left\{S_{i}^{\alpha}: i<n_{\alpha}\right\}$ the discrete topology and give $P=\prod_{\alpha<\kappa}\left\{S_{i}^{\alpha}: i<n_{\alpha}\right\}$ the product topology. Note that $w(P) \leqslant \kappa$.

Claim. There exists $S_{0} \in \mathcal{S}$ and a nonempty closed $C_{0} \subseteq S_{0}$ such that $f\left(S_{0}\right) \subseteq F$ and $f\left(C_{0}\right)$ is a $G_{\kappa}$ subset of $Y$.

Proof of Claim. Assume not. We will get a contradiction by constructing a $\kappa^{+}$ strictly decreasing sequence of closed sets of $P$. Define the (not necessarily continuous) function $\pi: \exp X \rightarrow \exp P$ by

$$
\pi(C)=\left\{\left(S_{\varphi(\alpha)}^{\alpha}\right): C \cap \bigcap_{\alpha<\kappa} S_{\varphi(\alpha)}^{\alpha} \neq \varnothing\right\}
$$

Set $F_{0}=X$. Assume we have constructed nonempty closed subsets $F_{\beta}$ of $X$ for all $\beta<\gamma<\kappa^{+}$such that

(1) $\left\{F_{\beta}: \beta<\gamma\right\}$ is a decreasing $\gamma$-sequence and $\left\{\pi\left(F_{\beta}\right): \beta<\gamma\right\}$ is a strictly decreasing $\gamma$-sequence,

(2) $f\left(F_{\beta}\right)$ is a $G_{\kappa}$ subset of $Y$.

If $\gamma$ is a limit ordinal, set $F_{\gamma}=\bigcap_{\beta<\gamma} F_{\beta}$. Since $f\left(F_{\gamma}\right)=f\left(\cap_{\beta<\gamma} F_{\beta}\right)=\bigcap_{\beta<\gamma} f\left(F_{\beta}\right)$ and each $f\left(F_{\beta}\right)$ is a $G_{\kappa}$ subset of $Y$, we have that $f\left(F_{\gamma}\right)$ is a $G_{\kappa}$ subset of $Y$. Also,

$$
\varnothing \neq \pi\left(F_{\gamma}\right)=\left\{\left(S_{\varphi(\alpha)}^{\alpha}\right): \bigcap_{\beta<\gamma} F_{\beta} \cap \bigcap_{\alpha<\kappa} S_{\varphi(\alpha)}^{\alpha} \neq \varnothing\right\}=\bigcap_{\beta<\gamma} \pi\left(F_{\beta}\right) \underset{+}{\subset} \pi\left(F_{\beta}\right)
$$

for all $\beta<\gamma$.

If $\gamma=\beta+1$, then choose a $\left(S_{\varphi(\alpha)}^{\alpha}\right) \in \pi\left(F_{\beta}\right)$. Therefore, $F_{\beta} \cap \bigcap_{\alpha<\kappa} S_{\varphi(\alpha)}^{\alpha} \neq \varnothing$. If $f\left(F_{\beta} \cap \cap_{\alpha<\kappa} S_{\varphi(\alpha)}^{\alpha}\right)=f\left(F_{\beta}\right)$, then the claim is true, for we can set $C_{0}=F_{\beta} \cap$ $\bigcap_{\alpha<\kappa} S_{\varphi(\alpha)}^{\alpha}$ and $S_{0}=\bigcap_{\alpha<\kappa} S_{\varphi(\alpha)}^{\alpha}$. Since we have assumed the claim is false, we can choose a nonempty zero set $Z$ of $f\left(F_{\beta}\right)$ such that $Z \subseteq f\left(F_{\beta}\right)-f\left(F_{\beta} \cap \cap_{\alpha<\kappa} S_{\varphi(\alpha)}^{\alpha}\right)$. Now set $F_{\gamma}=F_{\beta} \cap f^{-1}(Z)$. Since $f\left(F_{\gamma}\right)=Z$ and $Z$ is a $G_{\omega}$ subset of $f\left(F_{\beta}\right)$ we have that $f\left(F_{\gamma}\right)$ is a $G_{\kappa}$ subset of $Y$. Since $\left(S_{\varphi(\alpha)}^{\alpha}\right) \in \pi\left(F_{\beta}\right)-\pi\left(F_{\gamma}\right)$ we have that $\pi\left(F_{\gamma}\right) \subset \pi\left(F_{\beta}\right)$.

Now, the $\left\{\pi\left(F_{\gamma}\right): \gamma<\kappa^{+}\right\}$forms a $\kappa^{+}$strictly decreasing sequence of closed sets of $P$. Our claim is proven.

To prove the lemma, we repeatedly apply the claim. Given an $S_{0}$ and $C_{0}$ as in the claim, we consider $f \uparrow S_{0}: S_{0} \rightarrow f\left(S_{0}\right)$. Si $S_{0}$ is a closed subbase for $S_{0}$ and $\delta \uparrow S_{0}=$ $\left(\delta \uparrow S_{0}\right)^{\cap}$. Also $f\left(C_{0}\right)$ is a nonempty $G_{\kappa}$ subset of $Y$, hence also of $f\left(S_{0}\right)$. So, we have a new set-up for which we can apply the claim again. In this way, we get $S_{i}$ 's $\in \mathcal{S}$ and closed sets $C_{i} \subseteq S_{i}$ such that $S_{0} \supseteq S_{1} \supseteq S_{2} \supseteq \cdots$ and $f\left(S_{i+1}\right) \subseteq f\left(C_{i}\right) \subseteq f\left(S_{i}\right)$ with the $f\left(C_{i}\right)$ 's being $G_{\kappa}$ subsets of $Y$ and $f\left(S_{0}\right) \subseteq F$. Now, $f\left(\cap_{i<\omega} S_{i}\right)=\bigcap_{i<\omega} f\left(S_{i}\right)$ $=\bigcap_{i<\omega} f\left(C_{i}\right)$ which is a $G_{\kappa}$ subset of $Y$ contained in $F$. Setting $S=\bigcap_{i<\omega} S_{i}$ completes the proof of the lemma.

5. The hyperspace of $2^{\kappa+}$. If $\alpha<\kappa^{+}$and $F$ is a closed subset of $2^{\alpha}$, then we set $\hat{F}=\left\{H: H\right.$ is a closed subset of $2^{\kappa+}$ and $\left.H \uparrow \alpha=F\right\}$. Note that $\hat{F} \subseteq \exp 2^{\kappa+}$ and that if $\pi_{\alpha}: 2^{\kappa+} \rightarrow 2^{\alpha}$ is the projection map, then $\hat{F}=\left(\exp \pi_{\alpha}\right)^{-1}(F)$. We collect the following simple facts into a lemma. 
5.1. Lemma. Let $\alpha<\kappa^{+}, F$ a closed subset of $2^{\alpha}$ and $\widetilde{y}$ a closed $G_{\kappa}$ subset of $\exp 2^{\kappa+}$. Then,

(a) $\hat{F}$ is a closed $G_{\kappa}$ subset of $\exp 2^{\kappa+}$,

(b) $\hat{F} \rightarrow \exp F$,

(c) there exists $\beta<\kappa^{+}$such that $\bar{y}=\cup_{F \in \bar{F}}(F \uparrow \beta) \hat{\text {. }}$

Proof. (a) and (c) are well-known dyadic facts. For (b), define $\varphi: \hat{F} \rightarrow \exp F$ by $\varphi(H)=\{s \in F: \hat{s} 1 \in H \uparrow \alpha+1\}$.

6. $\exp 2^{\omega_{2}}$ is not superadic. For $\kappa$ a cardinal, set $P_{\kappa}=\cup_{\alpha<\kappa^{+}} \exp 2^{\alpha}$. If $F \in \exp 2^{\alpha}$ and $G \in \exp 2^{\beta}$, define $F \leqslant G$ if $\beta \leqslant \alpha$ and $F\left\lceil\beta=G\right.$. Then, $\left(P_{\kappa}, \leqslant\right)$ is a poset in which every decreasing $\kappa$-sequence has an infimum. We note that if $F \leqslant G$, then $F \rightarrow G$ by the projection map.

We are now ready to prove our main theorem.

6.1. THEOREM. Let $S=S^{\cap}$ be a closed subbase for a compact space $X$ and let $X \rightarrow \exp 2^{\kappa+}$. If $Y$ is a compact 0 -dimensional space of weight at most $\kappa$, then there exists an $S \in$ S such that $S \rightarrow \exp Y$.

Proof. Assume that $f: X \rightarrow \exp 2^{\kappa+}$ and that $Y$ is a closed subspace of $2^{\kappa}$. We will find an $F \leqslant Y$ and an $S \in \mathcal{S}$ such that $S \rightarrow \exp F$. Since $F \rightarrow Y$, we have $\exp F \rightarrow \exp Y$ and thus $S \rightarrow \exp Y$.

Put $S_{1}=X, \alpha_{1}=\kappa$ and $F_{\alpha_{1}}=Y$. Assume we have found $S_{n} \subseteq S_{n-1} \subseteq \cdots \subseteq S_{1}$ with all $S_{i} \in S$ and $\alpha_{1}<\alpha_{2}<\cdots<\alpha_{n}<\kappa^{+}$with $F_{\alpha_{1}} \in \exp 2^{\alpha_{i}}$ such that

(1) $\hat{F}_{\alpha_{i}} \subseteq f\left(S_{i}\right)$,

(2) $f\left(S_{i}\right)$ is a $G_{\kappa}$ subset of $\exp 2^{\kappa+}$.

(3) $S_{i} \subseteq S_{i-1} \cap f^{-1}\left(\hat{F}_{\alpha_{i-1}}\right)$, if $i \geqslant 2$.

At stage $n+1$, consider $f \uparrow S_{n}: S_{n} \rightarrow f\left(S_{n}\right)$. Then, $\mathcal{S} \uparrow S_{n}=\left(S \uparrow S_{n}\right)^{n}$ is a closed subbase for $S_{n}$. By Lemma 5.1(a), $\hat{F}_{\alpha_{n}}$ is a closed $G_{\kappa}$ subset of $\exp 2^{\kappa+}$ and thus $\hat{F}_{\alpha_{n}}$ is a closed $G_{\kappa}$ subset of $f\left(S_{n}\right)$. The Subbase Lemma now implies that there is a nonempty $S_{n+1} \in \mathcal{S} \mid S_{n}, S_{n+1} \subseteq S_{n}$ such that $f\left(S_{n+1}\right) \subseteq \hat{F}_{\alpha_{n}}$ and $f\left(S_{n+1}\right)$ is a $G_{\kappa}$ subset of $f\left(S_{n}\right)$. By inductive assumption (2), we conclude that $f\left(S_{n+1}\right)$ is a $G_{\kappa}$ subset of $\exp 2^{\kappa+}$. This verifies inductive assumptions (2) and (3) for $S_{n+1}$.

By Lemma 5.1(c), there exists $\alpha_{n+1}>\alpha_{n}$ such that

$$
f\left(S_{n+1}\right)=\bigcup_{F \in f\left(S_{n+1}\right)}\left(F \uparrow \alpha_{n+1}\right)^{\wedge} .
$$

Set $F_{\alpha_{n+1}}=H \uparrow \alpha_{n+1}$ for some $H \in f\left(S_{n+1}\right)$. Then, $\hat{F}_{\alpha_{n+1}} \subseteq f\left(S_{n+1}\right)$, verifying inductive assumption (1) for $F_{\alpha_{n+1}}$ and $S_{n+1}$.

Having completed the induction, we see that the $\hat{F}_{\alpha_{i}}$ 's are interlaced with the $f\left(S_{i}\right)$ 's, hence $\bigcap_{i<\omega} \hat{F}_{\alpha_{i}}=\bigcap_{i<\omega} f\left(S_{i}\right)$. If we set $F=\inf \left\{F_{\alpha_{i}}: i<\omega\right\}$, we have that $\hat{F}=\bigcap_{i<\omega} \hat{F}_{\alpha_{i}}$. Since $\bigcap_{i<\omega} f\left(S_{i}\right)=f\left(\cap_{i<\omega} S_{i}\right)$, by setting $S=\bigcap_{i<\omega} S_{i}$, we have that $S \in \mathcal{S}, \quad F \leqslant F_{\alpha_{1}}=Y$, and $S \rightarrow \hat{F}$. By Lemma 5.1(b), $\hat{F} \rightarrow \exp F$ and thus $S \rightarrow \exp F$, completing the proof.

6.2. Corollary. $\exp 2^{\omega_{2}}$ is not superadic. 
Proof. Assume there exists a space $X$ with a binary closed subbase $\delta$ (w.l.o.g. $\varsigma=\varsigma^{n}$ ) such that $X \rightarrow \exp 2^{\omega_{2}}$. Observe that if $S \in \mathcal{S}$, then $\delta \uparrow S$ is a binary closed subbase for $S$, hence $S$ is supercompact. Theorem 6.1 implies that if $Y$ is a compact 0 -dimensional space of weight $\omega_{1}$, then $\exp Y$ is superadic. This is not true, see Example 3.4 .

This corollary generalizes Šapiro's result [14] that $\exp 2^{\omega_{2}}$ is not dyadic. It also shows that one of the simplest types of supercompact space need not have a supercompact hyperspace. Whether exp $X$ supercompact implies $X$ supercompact is not known. Since Sirota [15] has shown that $\exp 2^{\omega_{1}}$ is homeomorphic to $2^{\omega_{1}}$, we get that the continuum hypothesis is equivalent to the supercompactness of $\exp 2$.

Similarly, it can be shown that $\exp I^{\omega_{2}}$ is not superadic where $I$ is the closed unit interval. We decided to prove the result for $\exp 2^{\omega_{2}}$ for reasons of simplicity. It then follows from a result of Efimov [8] that if $X$ has a closed subspace $F$ such that $F \rightarrow 2^{\omega_{2}}$, then $\exp X$ is not superadic.

Can $\exp 2^{\omega_{2}}$ be a continuous image of a space $X$ with cmpn $X<\infty$ ? We suspect not, but our technique breaks down because we do not have an example of a compact 0-dimensional space $Y$ (in ZFC) of weight $\omega_{1}$, such that $\exp Y$ (or for that matter $Y$ ) is not a continuous image of any space $X$ of finite compactness number. We mention that $\beta \omega$ is such an example, but it has weight $c$.

Jan van Mill has made the following interesting deduction.

6.3. Corollary. If $X$ is a compact 0 -dimensional space with $s(X)>\omega_{1}$, then $\exp (\exp X)$ is not superadic.

Proof. Let $D$ be a discrete subspace of $X$ of size $\omega_{2}$. For each $d \in D$ choose a clopen set $C(d)$ such that $C(d) \cap D=\{d\}$. For each $d \in D$, set $\tilde{d}=\{F \in$ $\exp X: F \cap C(d) \neq \varnothing\}$. Then $\{\tilde{d}: d \in D\}$ is an independent collection of clopen subsets of $\exp X$. Consequently, $\exp X \rightarrow 2^{\omega_{2}}$. Hence, $\exp (\exp X) \rightarrow \exp 2^{\omega_{2}}$ and thus, $\exp (\exp X)$ is not superadic.

6.4. ExAmple. A 0 -dimensional space $X$ with $\exp X$ superadic but $\exp (\exp X)$ not superadic: Let $X=\alpha \omega_{2}$. $\exp X$ is supercompact, see Example 3.5. Since $s(X)=\omega_{2}$, $\exp (\exp X)$ is not superadic.

\section{REFERENCES}

1. P. Alexandroff, Zur Theorie der topologischen Räume, C. R. (Doklady) Acad. Sci. URSS 11 (1936), 55-58.

2. M. G. Bell, A cellular constraint in supercompact Hausdorff spaces, Canad. J. Math. 30 (1978), $1144-1151$.

3. Compact ccc non-separable spaces of small weight, Topology Proc. 5 (1980), 11-25.

4., Two Boolean algebras with extreme cellular and compactness properties, preprint.

5. M. G. Bell and J. van Mill, The compactness number of a compact topological space. I, Fund. Math. 106 (1980), 163-173.

6. E. van Douwen, Mappings from hyperspaces and convergent sequences, preprint.

7. E. van Douwen and J. van Mill, Supercompact spaces, Topology Appl. 13 (1982), 21-32.

8. B. Efimov, On imbedding of Stone-Čech compactifications of discrete spaces in bicompacta, Soviet Math. Dokl. 10 (1969), 1391-1394.

9. J. de Groot, Supercompactness and superextensions, Contributions to Extension Theory of Topological Structures (Sympos. Berlin, 1967), Deutscher Verlag Wiss., Berlin, 1969, pp. 89-90. 
10. I. Juhász, Cardinal functions in topologl--ten years later, Math. Centre Tracts, No. 123, Mathematisch Centrum, Amsterdam, 1980.

11. J. van Mill, Supercompactness and Wallman spaces, Math. Centre Tracts, No. 85, Mathematisch Centrum, Amsterdam, 1977.

12. J. van Mill and C. F. Mills, A nonsupercompact image of a supercompact space. Houston J. Math. 5 (1979), 241-247.

13. S. Mrówka, Mazur theorem and m-adic spaces, Bull. Acad. Polon. Sci. Sér. Sci. Math., 18 (1970), 299-305.

14. L. B. Šapiro, The space of closed subsets of $D^{N_{2}}$ is not a dyadic bicompact, Soviet Math. Dokl. 17 (1976), 937-941.

15. S. Sirota, Spectral representation of spaces of closed subsets of bicompacta, Soviet Math. Dokl. 9 (1968), 997-1000.

16. L. Vietoris, Bereiche zweiter Ordnung, Monatsh. Math. Phys. 32 (1922), 258-280.

Department of Mathematics and Astronomy, University of Manitoba, Winnipeg, Manitoba, CANADA R3T 2N2 